\title{
An effective subgradient method for simultaneous restoration and segmentation of blurred images
}

\author{
T. Serezhnikova ${ }^{1,2}$ \\ ${ }^{1}$ Krasovsky Institute of Mathematics and Mechanics UB RAS \\ 2 Ural Federal University, Ekaterinburg, Russia \\ *e-mail: sti@imm.uran.ru
}

Key words: image restoration, denoising, segmentation, subgradient construction

The segmentation of blurred and noise images is of great importance. There have been several recent works to link the problems of image segmentation and image reconstraction. Here we describe the universal subgradient method for simultaneous restoration and segmentation of blurred and noise images. Our method is based on the universal subgradient construction.

Our universal subgradient contains both the brightness function and the brightness function gradient.

In the paper we demonstrate that our method is effective for simultaneous restorations and segmentations of blurred images. 